This item was submitted to Loughborough's Research Repository by the author.

Items in Figshare are protected by copyright, with all rights reserved, unless otherwise indicated.

\title{
Vortex qubit based on an annular Josephson junction containing a microshort
}

PLEASE CITE THE PUBLISHED VERSION

http://dx.doi.org/10.1103/PhysRevB.81.014506

PUBLISHER

(C) American Physical Society

VERSION

VoR (Version of Record)

LICENCE

CC BY-NC-ND 4.0

REPOSITORY RECORD

Price, A.N., A. Kemp, Dmitry R. Gulevich, F.V. Kusmartsev, and A.V. Ustinov. 2019. “Vortex Qubit Based on an Annular Josephson Junction Containing a Microshort". figshare. https://hdl.handle.net/2134/12761. 


\title{
Vortex qubit based on an annular Josephson junction containing a microshort
}

\author{
A. N. Price, ${ }^{1}$ A. Kemp, ${ }^{1}$ D. R. Gulevich, ${ }^{2}$ F. V. Kusmartsev, ${ }^{2}$ and A. V. Ustinov ${ }^{3}$ \\ ${ }^{1}$ Physikalisches Institut III, Universität Erlangen-Nürnberg, Erwin-Rommel-Str. 1, 91058 Erlangen, Germany \\ ${ }^{2}$ Department of Physics, Loughborough University, Loughborough LE11 3TU, United Kingdom \\ ${ }^{3}$ Physikalisches Institut, Universität Karlsruhe, Wolfgang-Gaede-Str. 1, 76131 Karlsruhe, Germany
}

(Received 28 October 2009; published 8 January 2010)

\begin{abstract}
We report theoretical and experimental work on the development of a vortex qubit based on a microshort in an annular Josephson junction. The microshort creates a potential barrier for the vortex, which produces a double-well potential under the application of an in-plane magnetic field; the field strength tunes the barrier height. A one-dimensional model for this system is presented, from which we calculate the vortex-depinning current and attempt frequency as well as the interwell coupling. Implementation of an effective microshort is achieved via a section of insulating barrier that is locally wider in the junction plane. Using a junction with this geometry we demonstrate classical state preparation and readout. The vortex is prepared in a given potential well by sending a series of "shaker" bias-current pulses through the junction. Readout is accomplished by measuring the vortex-depinning current.
\end{abstract}

DOI: 10.1103/PhysRevB.81.014506

PACS number(s): 03.67.Lx, 74.50.+r, 85.25.Cp

\section{INTRODUCTION}

Superconducting qubits based on Josephson junctions are one of the most promising qubit architectures in terms of scalability and ready integration with semiconductor electronics. Josephson junctions have been successfully utilized to build various types of qubits such as charge, ${ }^{1}$ phase,${ }^{2}$ and flux $^{3}$ qubits. The operation of these systems is based on quantum coherence of the charge state, the Josephson phase difference, or the magnetic-flux state, respectively. Elements characteristic of the charge and flux qubits are combined in a hybrid qubit called quantronium. ${ }^{4}$

By contrast with other types of superconducting qubits, a vortex qubit is designed to exploit the coherent superposition of two spatially separated states for a Josephson vortex within a long Josephson junction. ${ }^{5-7}$ These states correspond to the minima of a double-well potential. The landscape of potential energy experienced by the vortex along the length of the junction can be constructed as desired by spatially varying one or more of the following parameters: the junction barrier thickness and hence the critical current density, ${ }^{8-13}$ the magnitude of the in-plane magnetic field, ${ }^{7,14,15}$ the curvature of the junction centerline, ${ }^{5,6,16}$ and the width of the junction. ${ }^{17-19}$

In this paper, we report theoretical considerations and experimental results concerning a suggested vortex qubit ${ }^{8}$ which consists of a long one-dimensional annular junction ${ }^{20}$ containing a microshort. ${ }^{21}$ For this type of qubit, a quantitative fit between an analytical-model and experimental data in the classical regime is presented. The investigated system is schematized in Fig. 1. In a microshort qubit, competition between repulsion at the microshort and pinning by an inplane magnetic field ${ }^{9,22}$ creates a double-well potential for the vortex. ${ }^{8,12}$ In having a double-well potential, the microshort qubit resembles the flux qubit. ${ }^{23,24}$ An advantage of qubit basis states being localized in separate wells is that intrawell energy relaxation does not cause a false readout result. The quantum state of the microshort qubit is manipulated by applying a short pulse of magnetic field, which low- ers the height of the microshort-induced potential barrier and thus enables coherent oscillation between the basis states. This field plays the same role in controlling the tunneling amplitude as the external magnetic flux in the early charge qubit. ${ }^{25}$

We have enhanced a previously outlined design ${ }^{8}$ of the microshort qubit by implementing a microshort compatible with standard lithographic fabrication processes. ${ }^{19}$ At a "lithographic" microshort, the in-plane width of the tunnel barrier is fractionally larger than it is elsewhere in the junction, resulting in a locally enhanced critical current density per unit length. This planar structure aids the monolithic integration of microshort qubits in rapid single flux quantum (RSFQ) circuits, as does the junction being large enough for lithographic patterning and having a compatible criticalcurrent density $J_{C}$. With RSFQ logic as the interface between vortex qubits and room-temperature electronics, the circuits could readily be scaled up to the large numbers needed for

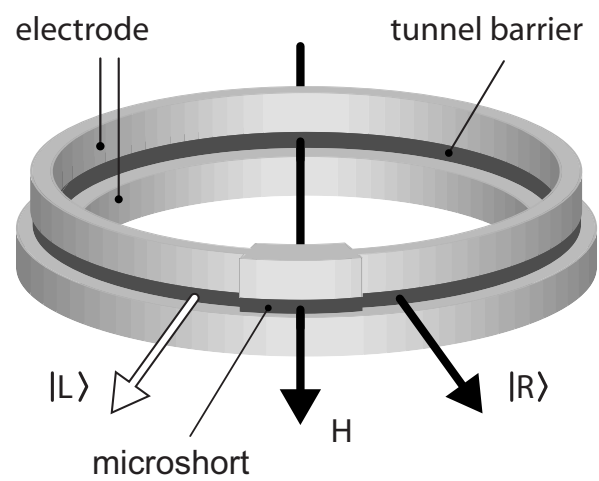

FIG. 1. A vortex qubit consisting of a one-dimensional annular junction with a microshort formed by a section of tunnel barrier of slightly greater in-plane width. Potential wells develop to the left $|L\rangle$ and right $|R\rangle$ of the microshort when a static magnetic field $H$, represented by the long arrow, is applied. A vortex at rest at either minimum is in a classically stable state. The magnetic moment of the vortex is indicated by a short arrow. (For clarity, the schematic is not drawn to scale.) 
useful computations. We have previously reported ${ }^{19}$ that the magnitude of the bias current required to drive a vortex past a lithographic microshort was an order of magnitude larger than expected. In this paper, we explain that the discrepancy is due to interaction between the lithographic microshort and magnetic field oriented transverse to the junction plane.

A number of characteristics of the proposed microshort qubit are tunable during experiment. The applied in-plane magnetic field controls the height of the potential barrier, the separation of the potential minima and the frequency of coherent oscillation of the vortex. Also the coupling between microshort qubits is adjustable. Josephson vortex qubits placed in a superconducting transmission line couple via virtual electromagnetic waves excited and absorbed by vortices. ${ }^{7}$ This indirect interaction depends on the tunneling amplitude of each qubit.

Since quantum tunneling of a single vortex out of a metastable potential well has been demonstrated, ${ }^{22}$ the next major step in realizing a vortex qubit is observing coherent oscillation of a vortex in a double-well potential. Using the experimentally investigated system detailed in this paper, we have observed a single vortex escaping from a metastable state by tunneling through a microshort-induced barrier. ${ }^{26}$ However we limit the experimental data presented here to the classical regime, focusing on state preparation and readout.

The theoretical section of this paper, Sec. II, begins with a derivation of the one-dimensional vortex potential for the proposed qubit. An analytical expression is obtained for the depinning current of a vortex over a microshort-induced potential barrier as a function of bias current and in-plane magnetic field strength. Also, the attempt frequency of the vortex in the presence of large magnetic field is derived for the cases of zero-bias current and bias just below the critical current. From the attempt frequency and the height of the potential barrier, we find the field dependence of the coupling between degenerate potential minima.

Experimental results obtained in the classical regime are presented in Secs. III and IV. We report measurements of the vortex-depinning current as a function of magnetic field strength that indicate the presence of bistable vortex states. Our readout scheme, based on the unique depinning current of each state, is described along with results which confirm that the vortex was prepared in a chosen initial state by means of shaker bias-current pulses. The final section, Sec. IV, discusses how the vortex-depinning current over a lithographic-microshort-induced potential barrier can be enhanced by flux trapped around both junction electrodes or magnetic field applied transverse to the junction plane.

\section{VORTEX IN AN ANNULAR MICROSHORT JUNCTION THEORY}

\section{A. One-dimensional model}

A homogeneous long Josephson junction is well described by the one-dimensional sine-Gordon equation for the phase difference $\varphi$ between the order parameters of the superconducting electrodes ${ }^{27,28}$

$$
\varphi^{\prime \prime}-\ddot{\varphi}-\sin \varphi=f .
$$

The spatial coordinate $x$ (see Fig. 2) is in units of the characteristic length scale, the Josephson length $\lambda_{J}$, while time $t$

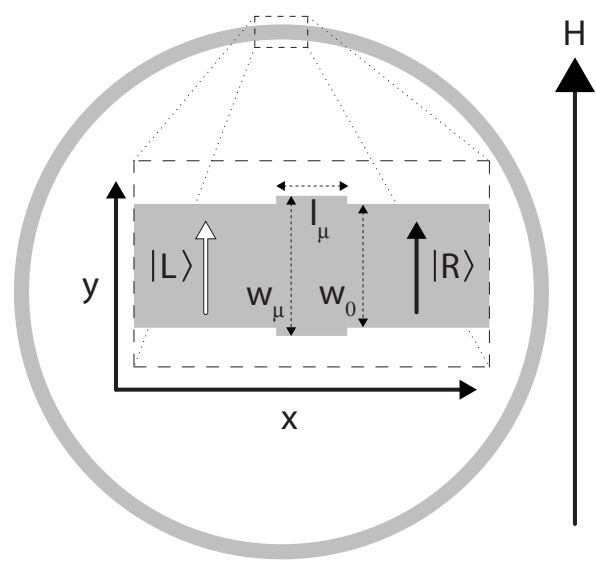

FIG. 2. Plan view of the tunnel-barrier layer of an annular junction with a lithographic microshort. The enlargement shows the effective microshort in more detail, where the barrier layer is fractionally wider than it is everywhere else in the junction.

is normalized to the inverse of the Josephson plasma frequency $\omega_{P}$. The perturbation

$$
f(x)=\gamma+\frac{\partial h_{R}}{\partial x}
$$

incorporates the bias current $\gamma$ and the current induced by the radial component $h_{R}$ of the normalized external magnetic field $h=\kappa H / H_{0}$. The bias current $\gamma$ is normalized to the product of the critical-current density $J_{C}$ and the junction area. The characteristic field is given by $H_{0}=\Phi_{0} / 2 \pi \mu_{0} d \lambda_{J}$ where $\Phi_{0}$ is the magnetic-flux quantum, $\mu_{0}$ is the vacuum permeability, $d$ is the magnetic thickness of the junction, and $\kappa$ is the geometric coupling factor between the magnetic field applied and that in the junction. For a linear junction $\partial h_{R} / \partial x$ $=0$ but in the annular case $\partial h_{R} / \partial x=-(h / r) \sin (x / r)$ where $r$ is the junction radius. Dissipative terms have not been included in Eq. (1) because, for the purposes of the experiments described in Secs. III and IV, the damping in our system at low temperatures is small enough to neglect.

In the absence of external field and applied bias current, one of the solutions of Eq. (1) is that of the stationary Josephson vortex

$$
\varphi^{v}=4 \arctan \left[\exp \left(x-x_{0}\right)\right] .
$$

The Josephson vortex is a topological soliton, behaving like a particle of normalized rest mass energy $m_{0}=8$ and centerof-mass position $x_{0}$. Here the unit of energy $\mathcal{E}_{0}$ $=J_{C} w_{0} \lambda_{J} \Phi_{0} / 2 \pi$ is the Josephson coupling energy of a small junction of area $w_{0} \lambda_{J}, w_{0}$ being the junction width.

To find the potential seen by a vortex in an annular junction, we begin with the Hamiltonian corresponding to Eq. (1)

$$
\mathcal{H}_{0}=\int_{0}^{l}\left(\frac{1}{2} \varphi^{\prime 2}+\frac{1}{2} \dot{\varphi}^{2}+1-\cos \varphi+f \varphi\right) d x .
$$

Approximating the spatial phase profile $\varphi(x)$ in Eq. (4) with Eq. (3), that of a vortex, and carrying out the integration over the entire length $l=2 \pi r$ of the junction, one obtains the washboard potential ${ }^{27}$ 


$$
U\left(x_{0}\right)=-2 \pi\left(\gamma x_{0}+h \operatorname{sech} \frac{\pi}{2 r} \cos \frac{x_{0}}{r}\right) .
$$

The first term $U^{\gamma}=-2 \pi \gamma x_{0}$ reflects the driving action of the bias current on the vortex while the second, $U^{h}=$ $-2 \pi h \operatorname{sech}(\pi / 2 r) \cos \left(x_{0} / r\right)$, originates in the convolution of the vortex spatial magnetic field profile $\partial \varphi_{v} / \partial x=2 \operatorname{sech} x$ with the applied field component $h_{R}$

$$
U^{h}=-h_{R} * \frac{\partial \varphi_{v}}{\partial x}
$$

Next we extend Eq. (5) by adding the contribution due to the lithographic microshort depicted in Fig. 2.

The characteristic energy scale $\mathcal{E}_{0}$ and hence the Josephson vortex rest-mass energy $m_{0}$ are proportional to the width of the junction. Thus one expects that a short length $l_{\mu}$ of broader junction will act as a potential barrier to the vortex, just as a microshort formed by decreasing the tunnel-barrier thickness would. In the case where the lithographicmicroshort length is smaller than the characteristic vortex size, $l_{\mu}<\lambda_{J}$, it is important to consider the spatial distribution of the vortex mass.

The spatial rest-mass profile is found from the sineGordon Hamiltonian for a static vortex: Eq. (4) with $f=0$. The time-independent phase distribution, Eq. (3), gives rise to equal magnetic field and Josephson coupling energy densities, $\varphi^{\prime 2} / 2$ and $1-\cos \varphi$, respectively. Hence the vortex rest-mass energy can be written as

$$
m_{0}=\int_{0}^{l} \tilde{\mu} d x
$$

where the spatial distribution of the vortex rest mass is

$$
\tilde{\mu}=4 \operatorname{sech}^{2}\left(x-x_{0}\right) .
$$

To calculate the change of vortex rest-mass energy caused by altering the junction width, we start with an appropriate Hamiltonian

$$
\mathcal{H}=\int_{0}^{l} \frac{w(x)}{w_{0}}\left(\frac{1}{2} \varphi^{\prime 2}+1-\cos \varphi\right) d x
$$

neglecting the perturbations due to bias current and external magnetic field. Note that the energy normalization $\mathcal{E}_{0}$ remains constant since the factor $w(x) / w_{0}$ describing the spatial variation in the junction width appears explicitly in Eq. (9). We only consider small width changes so the phase distribution $\varphi(x, y)=\varphi(x)$ remains radially independent. ${ }^{17}$ One now sees that the vortex rest-mass energy $m_{0}$ in a long junction of variable width consists of the convolution of the spatial rest-mass profile belonging to a uniform junction with the local potential energy per unit length of the variable width junction

$$
m_{0}=\tilde{\mu} * \frac{w}{w_{0}} .
$$

The kernel $\tilde{\mu} \sim \operatorname{sech}^{2}\left(x-x_{0}\right)$ of this convolution integral has a different shape to the magnetic field profile $\partial \varphi_{v} / \partial x$ $\sim \operatorname{sech}\left(x-x_{0}\right)$ in Eq. (6). The rest mass and magnetic field

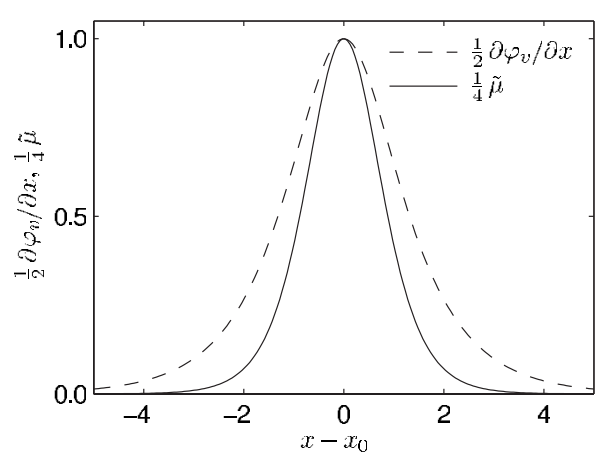

FIG. 3. Comparison of the kernels $\partial \varphi_{v} / \partial x$ and $\tilde{\mu}$ of the convolution integrals which, respectively, give rise to the magnetic field $U^{h}\left(x_{0}\right)$ and microshort $U^{\mu}\left(x_{0}\right)$ contributions to the vortex potential. The rest-mass profile $\tilde{\mu} \sim \operatorname{sech}^{2}\left(x-x_{0}\right)$ (solid line) of the vortex is spatially more tightly confined than its magnetic field profile $\partial \varphi_{v} / \partial x \sim \operatorname{sech}\left(x-x_{0}\right) \quad$ (dashed line); therefore the resultant microshort barrier tends to be narrower and the associated vortex quantum-tunneling rate should be enhanced (Ref. 13).

profiles are plotted together in Fig. 3; for the same amplitude, the rest-mass profile is narrower. This means that the potential barrier caused by a microshort tends to be more spatially confined than a barrier produced by an external field. Consequently, quantum tunneling of a Josephson vortex through a microshort-induced barrier is expected to be enhanced. ${ }^{13}$

Turning to the specific long junction geometry depicted in Fig. 2, the width variation is

$$
\frac{w(x)}{w_{0}}=1+\frac{\Delta w}{w_{0}} \Pi\left(x / l_{\mu}\right),
$$

where $\Pi(x)$ is the unit rectangle function and $\Delta w=w_{\mu}-w_{0}$ is the amount by which the junction width at the lithographic microshort, $w_{\mu}$, is larger than the junction width elsewhere. For short microshorts, $l_{\mu} \ll \lambda_{J}$, an equivalent representation using the Dirac delta function is

$$
\frac{w(x)}{w_{0}}=1+\frac{\Delta w}{w_{0}} l_{\mu} \delta(x) .
$$

Then the potential energy corresponding to the change in vortex rest-mass energy evaluates to

$$
U^{\mu}\left(x_{0}\right)=\mu \operatorname{sech}^{2} x_{0},
$$

where $\mu=4 l_{\mu} \Delta w / w_{0}$ measures the strength of the microshort. Combining Eqs. (13) and (5) gives the vortex potential for an annular junction containing a microshort

$$
U\left(x_{0}\right)=\mu \operatorname{sech}^{2} x_{0}-2 \pi\left(\gamma x_{0}+h \operatorname{sech} \frac{\pi}{2 r} \cos \frac{x_{0}}{r}\right) \text {. }
$$

\section{B. Double-well potential}

The vortex potential in the absence of bias current, Eq. (14) with $\gamma=0$, has two wells for magnetic fields in the range $0<\bar{h}<1$ where 

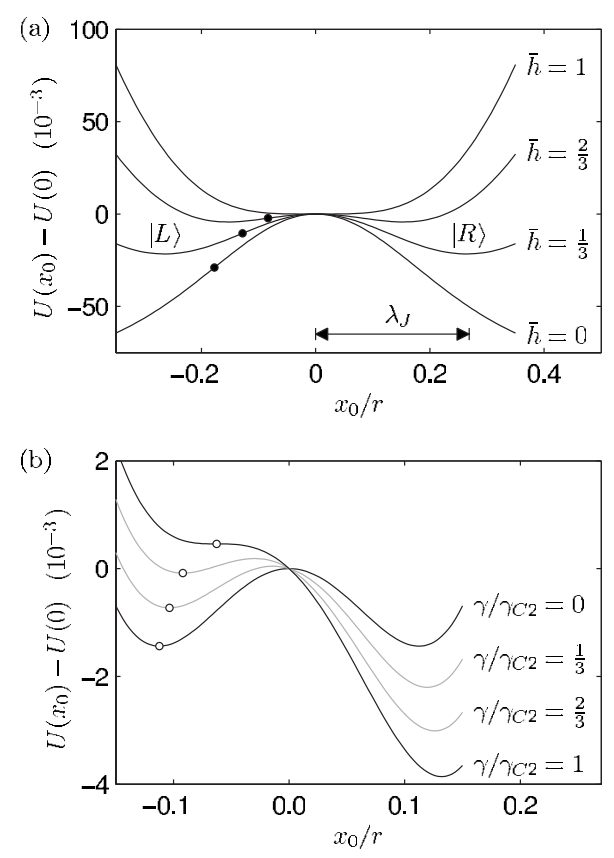

FIG. 4. The vortex potential $U\left(x_{0}\right)$ given by Eq. (14) for a long annular junction of radius $r=3.7$ containing a microshort of strength $\mu=0.09$. (a) The potential is plotted for four magnetic fields in the range $0 \leq \bar{h} \leq 1$ at zero-bias current. The double-well potential is tunable: as the magnetic field strength increases, both the height of the microshort-generated potential barrier and the physical separation between the stable vortex states $|L\rangle$ and $|R\rangle$ decrease. A black circle marks the location $x_{C 2}$ at which an inflection point forms under the critical bias $\gamma_{C 2}$. (b) The potential at $\bar{h}=0.8$ is shown for four bias currents in the range $0 \leq \gamma \leq \gamma_{C 2}$. A white circle indicates the location of the left minimum, which becomes an inflection point at $\gamma=\gamma_{C 2}$.

$$
\bar{h} \equiv \frac{h \pi \operatorname{sech}(\pi / 2 r)}{\mu r^{2}} .
$$

Figure 4(a) contains plots of this potential for various values of the magnetic field. The parameters $\mu, \lambda_{J}$, and $r$ were chosen to reflect the experimentally investigated system, which is detailed in Sec. III A. The height of the potential barrier shrinks with increasing magnetic field strength. In this way, the barrier height can be quickly controlled during experiment by, for example, passing current through an appropriately oriented microstrip underneath the junction. The field at which the barrier disappears completely is found by calculating when the curvature $U^{\prime \prime}\left(x_{0}\right)$ of the potential becomes positive at $x_{0}=0$. The left $|L\rangle$ and right $|R\rangle$ wells of the potential constitute stable classical states for the vortex.

The states are distinguished by their critical, or depinning, current. This is the amount of bias current needed for the vortex to overcome the pinning potential. We denote the depinning currents for the field- and microshort-induced barriers as $\gamma_{C 1}$ and $\gamma_{C 2}$, respectively. As shown in Fig. 4(b) for field $\bar{h}=0.8$, a larger bias current tilts the potential further. The left potential minimum, indicated by a white circle, turns into a horizontal inflection point at the critical bias $\gamma_{C 2}$. The location at which the inflection point develops, $x_{C 2}$, is repre- sented in Fig. 4(a) by black circles. The depinned vortex gains kinetic energy as it moves through the microshort and beyond in the direction of increasing $x$, and the junction voltage becomes nonzero. The vortex is metastable at bias currents slightly below that required to transform its potential well into an inflection point. This is due to thermal fluctuations and quantum tunneling, which reduce the observable critical current. At a large enough magnetic field $\bar{h}$, a vortex which has depinned from the left $|L\rangle$ well will be retrapped by the field-induced potential barrier before the switch to the resistive state can be observed. This can be avoided during readout of the qubit state by decreasing the field before the depinning current is measured.

We now consider the critical current $\gamma_{C 1}(h)$ associated with a vortex escaping over the potential barrier induced by the magnetic field. Increasing positive bias current moves the right minimum $|R\rangle$ and the vortex farther in the positive $x$ direction. At the critical bias, the vortex reaches the location $x_{C 1}=\pi r / 2$, where its magnetic moment is perpendicular to the applied field and the potential has a saddle point. Here the vortex depins and the bias accelerates it. In the case of a long annular junction with a weak microshort strength $\mu$ $\ll 1$, it is reasonable to neglect the influence of the microshort on this critical current. Therefore ${ }^{9}$

$$
\gamma_{C 1}(h) \simeq \frac{|h|}{r} \operatorname{sech} \frac{\pi}{2 r} .
$$

Next we derive the critical current $\gamma_{C 2}(h)$ due to the microshort. In this case, we assume $x_{0} \sim 0$ and replace the cosine term in Eq. (14) with its Maclaurin series up to $O\left(x_{0}^{2}\right)$, neglecting an additive constant

$$
U\left(x_{0}\right) \simeq \mu\left(\operatorname{sech}^{2} x_{0}+\bar{h} x_{0}^{2}\right)-2 \pi \gamma x_{0} .
$$

At the critical bias $\gamma_{C 2}$, the vortex depins at the location $x_{C 2}$ where the potential has a saddle point

$$
U^{\prime}\left(x_{C 2}\right)=0
$$

and

$$
U^{\prime \prime}\left(x_{C 2}\right)=0 .
$$

The condition given by Eq. (19) leads to a quadratic equation in $\operatorname{sech}^{2} x_{C 2}$ with the solution

$$
\begin{aligned}
\operatorname{sech}^{2} x_{C 2} & =\frac{1+\sqrt{1+3 \bar{h}}}{3} \equiv \tau_{1}(\bar{h}), \\
x_{C 2} & =-\operatorname{arcsech} \sqrt{\tau_{1}} .
\end{aligned}
$$

The minus sign in Eq. (21) reflects the case of a vortex which moves in the positive $x$ direction as it escapes over the microshort-induced potential barrier. A larger applied field in the range $0<\bar{h}<1$ results in a depinning location $x_{C 2}$ which is nearer to the microshort center at $x=0$. After substituting Eq. (21) into Eq. (18), one obtains the field-dependent critical current for a vortex trapped by a microshort

$$
\gamma_{C 2}(h)=\frac{\mu}{\pi}\left(\tau_{1} \sqrt{1-\tau_{1}}-\bar{h} \operatorname{arcsech} \sqrt{\tau_{1}}\right) .
$$




\section{Attempt frequency}

A vortex in a potential well oscillates about its average position with frequency $\omega_{0}$, known as the small amplitude oscillation frequency. This is the frequency with which the vortex attempts to escape from the well; in a junction with moderate damping it is proportional to the rate of thermal escape [See Eq. (42)]. In the quantum regime, the energy $\hbar \omega_{0}$ separates the first excited state of the vortex from its ground state within a single well. For a symmetric doublewell potential, the frequency of coherent oscillation of the vortex between the $|L\rangle$ and $|R\rangle$ states, $\Delta_{0}$, depends exponentially on the small oscillation frequency $\omega_{0}$ [as seen from Eqs. (36) and (37)]. In this section, we derive analytical expressions for the attempt frequency $\omega_{0}$ at large fields $\bar{h} \lesssim 1$.

The frequency of attempted vortex escape varies as the square root of the curvature at the potential minimum

$$
\omega_{0}=\sqrt{\frac{U^{\prime \prime}\left(x_{1}\right)}{m_{0}}} .
$$

The location of the potential minimum is found by solving

$$
U^{\prime}\left(x_{i}\right)=0
$$

using the approximation $x \simeq \tanh x+\tanh ^{3} x / 3$, which is valid for small $x_{i}$ and therefore large normalized fields $\bar{h}$. The approximation leads to an analytically solvable cubic equation in $\tanh x_{i}$

$$
\tanh ^{3} x_{i}+\tau_{2} \tanh x_{i}=\tau_{3},
$$

where, for clarity, we define

$$
\tau_{2}(\bar{h}) \equiv-\frac{1-\bar{h}}{1+\bar{h} / 3} \quad \text { and } \quad \tau_{3}(\bar{h}) \equiv \frac{\pi \gamma}{\mu(1+\bar{h} / 3)} .
$$

The discriminant of the cubic equation, $D=\left(\tau_{2} / 3\right)^{3}+\left(\tau_{3} / 2\right)^{2}$, equals zero at the critical bias $\gamma_{C 2}$, where all roots are real and two are equal. From this, one obtains an approximation to Eq. (22)

$$
\gamma_{C 2}(\bar{h}) \simeq \frac{2 \mu(1-\bar{h})^{3 / 2}}{3 \sqrt{3} \pi(1+\bar{h})^{1 / 2}},
$$

whose relative error is under $0.7 \%$ over the range of magnetic fields for which the double-well potential exists. Defining the normalized bias current as $\bar{\gamma}=\gamma / \gamma_{C 2}(h)$ with $\gamma_{C 2}(h)$ given by Eq. (27) enables the solutions to Eq. (25) to be written as

$$
\tanh x_{i}=2 \sqrt{\frac{-\tau_{2}}{3}} \cos \left(\frac{\arccos \bar{\gamma}}{3}+\frac{2 n \pi}{3}\right) .
$$

The constant $n$ takes on values from the set $\{0,1,2\}$ for a double-well potential where the microshort is centered at $x$ $=0$. The set elements correspond, respectively, to the right potential minimum $|R\rangle$ at $x_{3}$, the left minimum $|L\rangle$ at $x_{1}$, and the potential maximum at $x_{2}$.

An analytical expression for the attempt frequency is found in the case of zero-bias current; substituting Eq. (28) with $\bar{\gamma}=0$ and $n=1$ into Eq. (23) leads to

$$
\omega_{0}=\frac{\sqrt{\mu \bar{h}(1-\bar{h})(33-\bar{h})}}{2(3+\bar{h})} .
$$

For large fields $\bar{h} \lesssim 1$, near the field at which the microshortinduced potential barrier disappears, a series expansion of the square of Eq. (29) about $\bar{h}=1$ reveals that the attempt frequency behaves as

$$
\omega_{0} \simeq \sqrt{\mu \epsilon_{h} / 2}
$$

where $\epsilon_{h} \equiv 1-\bar{h}$.

The effect of bias currents just below the critical current, $\bar{\gamma} \lesssim 1$, on the attempt frequency is of interest when interpreting microwave spectroscopy data for a vortex in a metastable state. Such data provide information on the shape of the potential well. For large magnetic fields, $\bar{h} \lesssim 1$, and large bias currents the curvature of the potential minimum can be described by

$$
U^{\prime \prime}\left(x_{1}\right) \simeq 4 \mu \epsilon_{h} \sqrt{2 \epsilon_{\gamma} / 3},
$$

where $\epsilon_{\gamma} \equiv 1-\bar{\gamma}$. On inserting Eq. (31) into Eq. (23), one finds that the attempt frequency for a vortex pinned by a microshort in a metastable potential well depends on the bias current as

$$
\omega_{0} \simeq \sqrt{\mu \epsilon_{h}}\left(\epsilon_{\gamma} / 6\right)^{1 / 4} .
$$

Just as for a vortex pinned by a microresistor in the absence of magnetic field, ${ }^{9,13}$ the small oscillation frequency $\omega_{0}$ varies with bias $\bar{\gamma}$ as the fourth root of the term $1-\bar{\gamma}$.

\section{Quantum properties}

To investigate the quantum properties of an annular Josephson junction with a lithographic microshort, we start with the normalized Euclidean action $S$, in units of $\mathcal{E}_{0} / \omega_{P}$, at zero temperature

$$
\begin{aligned}
S[\varphi(x, \tau)]= & \int_{0}^{\infty} d \tau \int_{-l / 2}^{l / 2} d x\left\{\frac{\varphi_{\tau}^{2}}{2}+\left[1+\frac{\mu}{4} \delta(x)\right]\left(\frac{\varphi_{x}^{2}}{2}+1\right.\right. \\
& -\cos \varphi)+f \varphi\} .
\end{aligned}
$$

Here the time variable is transformed as $t=i \tau$ and the vortex coordinate $x_{0}(\tau)$ is time dependent

$$
\varphi_{v}(x, \tau)=4 \arctan \left\{\exp \left[x-x_{0}(\tau)\right]\right\} .
$$

After integration of Eq. (33) over the spatial coordinate $x$, the effective action has the form

$$
S[\varphi(x, \tau)]=\int_{0}^{\infty} d \tau\left[\frac{m_{0} \dot{x}_{0}^{2}}{2}+U\left(x_{0}\right)\right],
$$

where $U\left(x_{0}\right)$ is given by Eq. (17).

At zero-bias current, the tunnel splitting $\hbar \Delta_{0}$ depends on the instanton action $S$ as ${ }^{29}$ 


$$
\hbar \Delta_{0}=4 \omega_{0} \hbar \sqrt{\frac{3 S}{2 \pi \hbar}} \exp \left(-\frac{S}{\hbar}\right)
$$

where

$$
S=\frac{16 \Delta U}{3 \omega_{0}}
$$

and $\Delta U$ denotes the height of the potential barrier. From Eqs. (17) and (28) with $n=1$

$$
\begin{aligned}
& \Delta U=\mu\left(\tanh ^{2} x_{1}-\bar{h} x_{1}^{2}\right), \\
& \simeq 3 \mu \epsilon_{h}^{2} / 8 \text { for } \bar{h} \lesssim 1 .
\end{aligned}
$$

Hence the action varies with large magnetic fields $\bar{h}$ as

$$
S=2 \sqrt{2 \mu} \epsilon_{h}^{3 / 2}
$$

and the quantum tunneling rate $\Delta_{0}$, in units of $\omega_{P}$, is given by

$$
\Delta_{0}=2 \sqrt{\frac{3}{\pi \hbar}}(2 \mu)^{3 / 4} \epsilon_{h}^{5 / 4} \exp \left(-\frac{2 \sqrt{2 \mu} \epsilon_{h}^{3 / 2}}{\hbar}\right) .
$$

\section{E. Operation as a two-level system}

Coherent oscillation of the vortex between the basis states $|L\rangle$ and $|R\rangle$ is controlled by short pulses of the in-plane field $H$. At zero-bias current, these states are degenerate. Oscillation takes place during a positive pulse, when the potential barrier $\Delta U$ is lower and the interwell coupling $\Delta_{0}$ is thereby increased. At this field, the potential minima are close enough to each other that the crossover temperature $T_{0}$, below which exponential relaxation gives way to underdamped coherent oscillation, is greater than the temperature $T$ of the qubit. ${ }^{30}$ After the end of the pulse, the crossover temperature $T_{0}$ is lower than the qubit temperature $T$, and the oscillation has ceased. Readout should take place before overdamped relaxation causes the vortex to transition to the other well. At this point, there is enough time to further lower the field if it is necessary to reduce the barrier height before the depinning current of the $|L\rangle$ state can be measured.

We now consider the feasibility of experiments in the quantum regime using realistic trilayer fabrication parameters. We assume a critical-current density of $J_{C}$ $=1 \mathrm{kA} / \mathrm{cm}^{2}$, a junction width of $w_{0}=0.6 \mu \mathrm{m}$, and an additional junction area of $\left(w_{\mu}-w_{0}\right) l_{\mu}=0.2 \mu \mathrm{m}^{2}$. This corresponds to a Josephson length of $\lambda_{J} \approx 12 \mu \mathrm{m}$, a microshort strength of $\mu=0.1$, and a reduced Planck's constant of $\hbar$ $=3.5 \times 10^{-3} \mathcal{E}_{0} / \omega_{P}$. In normalized units, the magnitude of the reduced Planck's constant $\hbar$ is inversely proportional to the junction width $w_{0}$. Hence quantum effects are more readily measured in narrower junctions. ${ }^{31}$ The length of the junction is taken to be $30 \lambda_{J}$.

We estimate an upper limit for the thermal escape rate $\Gamma_{\text {th }}$ by assuming moderate damping

$$
\Gamma_{\text {th }}=\frac{\omega_{0}}{2 \pi} \exp \left(-\frac{\Delta U}{k_{B} T}\right) .
$$

The actual thermal-activation rate is expected to be substantially slower because the junction will be in the low-damping

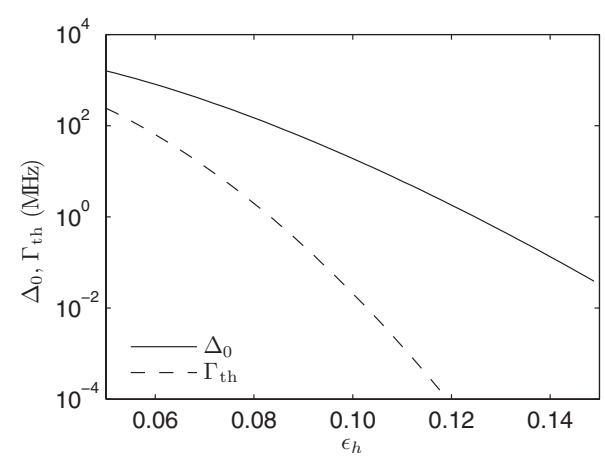

FIG. 5. Quantum-tunneling rate $\Delta_{0}$ (solid line) and upper limit for the thermal-activation rate $\Gamma_{\text {th }}$ (dashed line) for a junction of critical-current density $J_{C}=1 \mathrm{kA} / \mathrm{cm}^{2}$, width $w_{0}=0.6 \mu \mathrm{m}$, and microshort strength $\mu=0.1$.

regime. Figure 5 aids comparison of the upper limit for the thermal escape rate $\Gamma_{\text {th }}$ at temperature $T=50 \mathrm{mK}$ with the quantum-tunneling rate $\Delta_{0}$ given by Eq. (41). Clearly, thermal activation should be insignificant during operation of the junction as a two-level system at $T=50 \mathrm{mK}$.

The friction coefficient $\eta$ plays a crucial role in the dissipative dynamics of a two-level system. It is given by

$$
\eta=\frac{1}{\omega_{P} R C}
$$

where $C$ denotes the junction capacitance and $R=50 \Omega$, the real part of the impedance of the microstrip biasing lines feeding the junction. For our chosen junction parameters, the classical friction coefficient is $\eta=2 \times 10^{-3}$.

To determine the experimental conditions under which coherent oscillation of the vortex between the left $|L\rangle$ and right $|R\rangle$ potential minima is observable, we use the dimensionless system-environment coupling strength

$$
\alpha=\frac{\eta(\Delta x)^{2}}{2 \pi \hbar},
$$

where $\Delta x$ is the distance between the minima according to Eq. (28). The crossover temperature $T_{0}$ depends on the coupling strength $\alpha$ as ${ }^{30}$

$$
T_{0}=\frac{\hbar}{k_{B}} \frac{\Delta_{0}}{\alpha \pi} .
$$

Figure 6 displays the crossover temperature $T_{0}$, the barrier height $\Delta U$, and the spacing $\hbar \omega_{0}$ between the first excited state and the ground state in each well. At a temperature of $T=50 \mathrm{mK}$, an applied field of $\epsilon_{h} \sim 0.06$ for the quantum operation and $\epsilon_{h} \sim 0.14$ for the readout should yield a measurable quantum-tunneling rate of $\Delta_{0} \sim 700 \mathrm{MHz}$ and allow a readout time of up to tens of milliseconds. Rectangular field pulses of the required height $\Delta \bar{h}=0.08$ and frequency could be produced using a microstrip or the near field of an rf antenna. During quantum operation at $\epsilon_{h} \sim 0.06$, the level spacing $\hbar \omega_{0}$ is one order of magnitude greater than the tunnel splitting $\hbar \Delta_{0}$. Thus pulse frequencies comparable to the tunneling rate $\Delta_{0}$ can be used without excitation of the vortex 


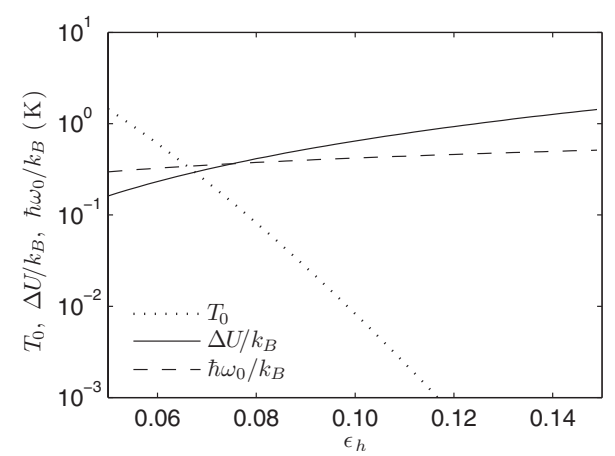

FIG. 6. Barrier height $\Delta U$ (solid line); spacing $\hbar \omega_{0}$ (dashed line) between the first excited state and the ground state in each well; and crossover temperature $T_{0}$ (dotted line), below which exponential relaxation becomes underdamped coherent oscillation. The junction parameters are the same as for Fig. 5.

to a higher level provided that the rise time of the field pulse is longer than $2 \pi / \omega_{0}$.

\section{EXPERIMENT}

\section{A. Samples and setup}

The results presented here pertain to two nominally identical long Josephson junctions of the geometry depicted in Fig. 2. The junctions were fabricated on different chips within the same run of a standard $\mathrm{Nb}-\mathrm{AlO}_{x}-\mathrm{Nb}$ trilayer process $^{32}$ and had a critical-current density of $J_{C}$ $=1.2 \mathrm{kA} / \mathrm{cm}^{2}$ at temperature $T=1.4 \mathrm{~K}$, which corresponds to a Josephson length of $\lambda_{J}=13 \mu \mathrm{m}$ (after including the effect of the idle region). ${ }^{33}$ The basic shape of the junctions was annular with a radius of $r=50 \mu \mathrm{m}$ and a width of $w_{0}$ $=2.4 \mu \mathrm{m}$. A short length $l_{\mu}=1.4 \mu \mathrm{m}$ of each junction was wider, $w_{\mu}=2.9 \mu \mathrm{m}$, forming a lithographic microshort of expected strength $\mu=0.09$. The junctions had Lyngby style ${ }^{20}$ electrode leads, whose width slightly exceeds the junction diameter $2 r$.

Each sample was situated within a solenoid which produced the in-plane magnetic field $H$. For measurements of the vortex-depinning current $I_{C}(H)$ at $T=1 \mathrm{~K}$, the solenoid was attached to the cold finger of a dilution refrigerator. The junction was biased through low-pass RC filters at the $1 \mathrm{~K}$ stage. ${ }^{34}$ Measurements at $T=1.4 \mathrm{~K}$ were carried out in a ${ }^{4} \mathrm{He}$ cryostat. For both setups, room-temperature feedthrough capacitor filters were used. The Josephson vortex was trapped upon cooling through the critical temperature of the niobium electrodes. Its depinning current was evaluated as the product of the known bias current ramp rate with the time elapsed between the ramp passing through zero current and the appearance of voltage across the junction electrodes. ${ }^{34}$

\section{B. Bistable states}

Measured data on the dependence of the vortex-depinning current $I_{C}$ as a function of the external magnetic field strength $H$ are plotted in Fig. 7 together with Eqs. (16) and (22). The $I_{C}(H)$ data consists of lines which are labeled according to the source of the potential barrier over which the



FIG. 7. Vortex-depinning current $I_{C}$ for junction 1 as a function of applied magnetic field strength $H$ at temperature $T=1.4 \mathrm{~K}$. The standard deviation, which is on the order of $1 \mu \mathrm{A}$, is not shown. Sections of the data (black circles) form approximately straight lines which originate in the escape of the vortex over a potential barrier induced by either (i) the magnetic field, (ii) the microshort, or (iii) the injectors (Refs. 35 and 36). The depinning currents expected from Eqs. (16) and (22) for the magnetic field and microshort barriers are also plotted (solid and dot-dashed lines, respectively). In calculating the depinning current from the magnetic field barrier, the self-field of the bias current was taken into account. The inset displays the supercurrent maxima of the $I_{C}(H)$ pattern as well as branches due to the nucleation of (vi) one or (v) two vortexantivortex pairs.

vortex escaped: (i) the magnetic field, (ii) the lithographic microshort, and (iii) the injectors. ${ }^{35,36}$ The branches which appear at high field $H$ originate from the nucleation of (vi) one or (v) two vortex-antivortex pairs. ${ }^{37}$ The injectors are leads for local current injection, which provide a means of inserting a vortex into the junction on demand. They were not used in the experimental work described in this paper and they do not affect the shape of the relevant part of the double-well potential, even though the injector branch is present at weak positive fields $H$. This is because the currentinjection leads are located at $x=\pi r$, as far from the microshort as possible.

The dependence of the depinning current on the magnetic field barrier is typical for long angular junctions. At zero field the barrier is absent and the junction critical current, at which the vortex depins, is therefore minimal. Increasing the magnetic field strength leads to a larger vortex-depinning current as expected from Eq. (16), which is plotted as a solid line in Fig. 7. In order to graph Eq. (16), the geometrical parameter $\kappa$ was calculated from the gradient of the branches labeled $i$ while taking into account the self-field of the bias current. The maximum critical current was measured independently and the values of the other junction parameters 
came from the specifications of the fabrication process.

Linear extrapolations of branch $i$ for positive and negative field $H$ do not intersect at zero critical current. This could be due to the self-field of a current induced by a small magnetic field $H_{Z}$ oriented perpendicular to the junction plane. The induced current circulates along the electrode rings. At $x_{0}=$ $+\pi r / 2$ and $x_{0}=-\pi r / 2$, where the vortex depins from the barrier induced by positive and negative field $H$, respectively, the self-field of the circulating current is of opposite polarity. The contribution of this self-field to the total inplane field $H$ shifts the measured branches $i$, for both positive and negative fields $H$, along the $H$ axis toward the origin.

For negative field polarity, the magnetic field barrier is centered at the microshort, at $x_{0}=0$, which results in a singlewell potential. For positive polarity, the magnetic field barrier, at $x_{0}=\pi r$, is located diametrically opposite the microshort barrier; together they form the double-well potential. As seen in Fig. 7, at temperature $T=1.4 \mathrm{~K}$ the stable vortex states of the double-well potential are distinguishable by means of the depinning current over the field interval $0 \leqq H \lesssim 5 \mathrm{~A} / \mathrm{m}$. Depinning currents from both of the two branches (i) and (ii) are also measured for fields near $H=-4 \mathrm{~A} / \mathrm{m}$ although the potential at zero bias consists of a single well. In this case the junction radius was large enough that, at finite bias, a second local potential minimum formed between the positions where the respective gradients of the field and microshort contributions to the potential, $\partial U^{h} / \partial x$ and $\partial U^{\mu} / \partial x$, were maximal.

In Fig. 7, the measured depinning current from the microshort barrier is several times larger than predicted by Eq. (22), which is represented by a dot-dashed line. We discuss this issue in Sec. IV B, where we show how an external magnetic field $H_{Z}$ perpendicular to the junction plane can result in an effective microshort strength $\bar{\mu}$ which grows with increasing field strength $H_{Z}$. When $\bar{\mu} \neq \mu$, the dependence of the vortex small oscillation frequency $\omega_{0}$ on the in-plane field $h$ differs from Eqs. (29) and (30). This prevents direct comparison of the data in Figs. 6 and 7. One means of avoiding interaction between the microshort and transverse field $H_{Z}$ is suggested in Sec. IV C.

\section{State preparation}

To base a quantum bit on this junction design, it is necessary to be able to produce a defined initial state. We prepared the vortex as desired in the left $|L\rangle$ or right $|R\rangle$ potential well by means of a shaker ${ }^{38}$ bias-current sequence, ${ }^{19}$ which is depicted in Fig. 8(a). The amplitude $\left|I_{P}\right|$ of the preparation pulses is such that a pulse frees the vortex only when it is pinned by the magnetic field and not when it is pinned by the microshort. The vortex remains pinned in the left $|L\rangle$ and right $|R\rangle$ wells for pulses of positive and negative polarity, respectively. With each successive pulse, the likelihood of locating the vortex in the chosen well increases. The number of pulses needed to reliably attain the desired state depends on the probability of the vortex being retrapped in the chosen well. Note that the vortex can also be prepared deterministically in the left $|L\rangle$ or right $|R\rangle$ well by applying
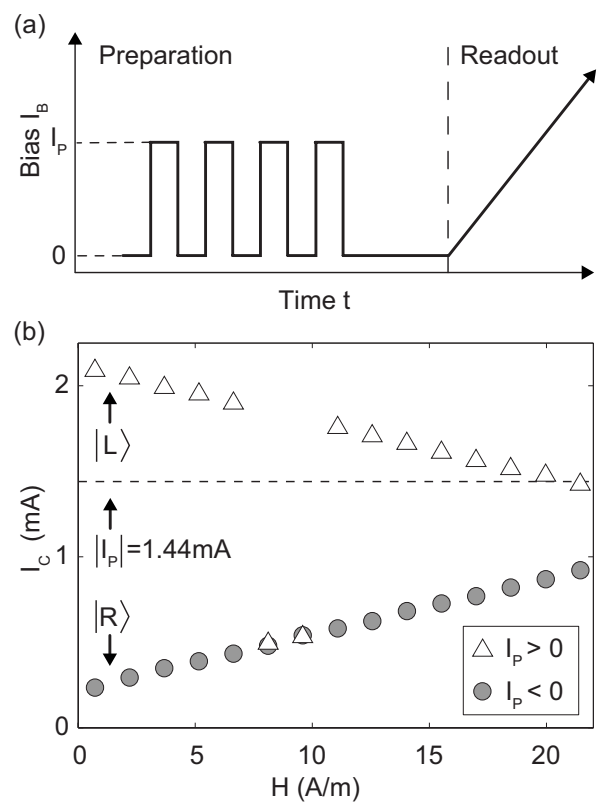

FIG. 8. State preparation by means of a series of shaker (Ref. 38) bias current pulses applied to junction 2 at temperature $T$ $=1 \mathrm{~K}$ (Ref. 19). (a) The bias current during state preparation and readout. (b) Positive (white triangles) and negative (gray circles) preparation pulses place the vortex in the left $|L\rangle$ and right $|R\rangle$ potential wells, respectively. The magnitude of the preparation pulses (dashed line) is indicated.

a bias pulse of the appropriate polarity at zero field and increasing the external field afterward but this procedure is slower due to solenoid inductance.

After the preparation stage, the bias current is increased at constant positive rate in order to measure the vortexdepinning current and identify which potential well the resting vortex was located in. The readout results ${ }^{19}$ for 30 preparation pulses of amplitude $\left|I_{P}\right|=1.44 \mathrm{~mA}$ in junction 2 at temperature $T=1 \mathrm{~K}$ are plotted in Fig. 8(b). The white triangles denote depinning currents measured after preparing the state with positive polarity pulses, and the gray circles, negative polarity pulses.

We observed over tens of trials that 30 preparation pulses are enough to prepare a given state at nearly all external magnetic field strengths. An exception occurred near $10 \mathrm{~A} / \mathrm{m}$ where the vortex could not be prepared in the left well $|L\rangle$ due to the low probability for it to be retrapped there. We found that the probability for the vortex to be retrapped in a particular well is temperature and field dependent. The temperature dependence of the retrapping probability may be caused by the damping changing with temperature. Initialization at a field $H$ where the probability of retrapping the vortex in the desired well is high offers the advantage that fewer preparation pulses are required. We expect that the shaker initialization process can be used at most fields where the depinning currents from the $|L\rangle$ or $|R\rangle$ wells are distinct as long as the amplitude $\left|I_{P}\right|$ of the preparation pulses lies between these currents and is greater than the depinning current associated with any other potential barriers in the junction. Therefore junction 1 could be initialized with this procedure over a field range from $H=0 \mathrm{~A} / \mathrm{m}$ to $H \sim 5 \mathrm{~A} / \mathrm{m}$, a shorter interval than for junction 2 . 


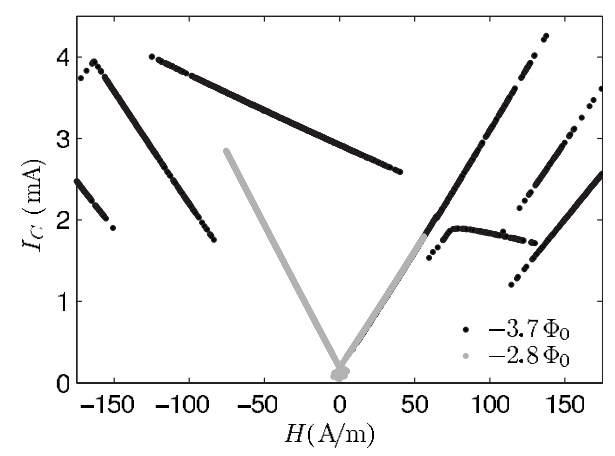

FIG. 9. Vortex-depinning current $I_{C}$ versus in-plane magnetic field $H$ at $T=1.4 \mathrm{~K}$, obtained after field cooling junction 1 under transverse magnetic fields equivalent to -2.8 (gray) and -3.7 (black) flux quanta through each electrode loop. The perpendicular field was switched off before the $I_{C}(H)$ pattern was recorded.

In Fig. 8(b), the measured depinning current from the left well $|L\rangle$, where the vortex is pinned by the microshort, is an order of magnitude greater than predicted by Eq. (22). Such a large enhancement occurs when magnetic flux is trapped around both electrodes. The flux-induced supercurrent interacts with the lithographic microshort as described in the next section.

\section{DISCUSSION}

\section{A. Enhanced vortex pinning due to flux trapped around both electrodes}

We have found that the vortex-depinning current strongly depends on the strength of a magnetic field $H_{Z}$ applied perpendicular to the junction plane during cooling below the superconducting transition temperature $T_{C} \sim 9.2 \mathrm{~K}$. After field cooling the transverse field $H_{Z}$ was switched off and then the critical current $I_{C}$ was measured as a function of the in-plane field $H$. We repeated this process for a range of transverse fields $H_{Z}$ and obtained several distinct $I_{C}(H)$ patterns. Each $I_{C}(H)$ pattern was observed for a particular subinterval of transverse field. Reversing the polarity of the applied transverse field reflected the $I_{C}(H)$ patterns about the $I_{C}$ and $H$ axes simultaneously. Further results pertaining to field-cooling annular junctions under perpendicular fields are presented elsewhere. ${ }^{39,40}$

Figure 9 shows $I_{C}(H)$ patterns which were obtained after field cooling under transverse fields $H_{Z}$ differing by around $0.2 \mathrm{~A} / \mathrm{m}$ and equivalent to fluxes of $\Phi_{Z}=-2.8 \Phi_{0}$ (gray) and $\Phi_{Z}=-3.7 \Phi_{0}$ (black) through each electrode loop. Whereas the $I_{C}(H)$ pattern obtained after field cooling under $\Phi_{Z}=$ $-2.8 \Phi_{0}$ is typical of a single trapped vortex, the pattern for $\Phi_{Z}=-3.7 \Phi_{0}$ resembles Fig. 8(b) in that the vortexdepinning current over the microshort-induced barrier is enhanced by an order of magnitude. The difference between these two $I_{C}(H)$ patterns originates in the amount of flux trapped within both electrode rings. Note that the microshort depinning current measured after field cooling under $\Phi_{Z}=$ $-2.8 \Phi_{0}$ does not agree with Eq. (22), possibly due to the influence of a weak background transverse field.
Field transverse to the junction plane induces a supercurrent $I_{\Phi}$ which circulates around the electrode loop. The fraction of supercurrent in each electrode is in the inverse ratio of their inductance per unit length. Due to the fabrication process, this ratio changes at the microshort boundaries, where not only the active region but also the neighboring part of the upper electrode widens as illustrated in Fig. 1. Consequently, some supercurrent $\varepsilon$ passes upward through the junction barrier on one side of the microshort and downward on the other. The corresponding spatial dependence of the Josephson phase $\varphi(x)$ is equivalent to that of a current dipole $^{35,36}$ or fractional vortex, ${ }^{41,42}$ objects which can increase the vortex-depinning current over the microshortinduced potential barrier. For a pointlike $\left(l_{\mu} \ll \lambda_{J}\right)$ dipole of strength $\kappa_{\mu}=\varepsilon l_{\mu}$, Eq. (2) becomes ${ }^{36}$

$$
f(x)=\gamma+\frac{\partial h_{R}}{\partial x}+\kappa_{\mu} \delta^{\prime}(x) .
$$

The contribution of the lithographic microshort to the vortex potential is then

$$
\begin{aligned}
& U^{\mu}\left(x_{0}\right)=\mu \operatorname{sech}^{2} x_{0}-2 \kappa_{\mu} \operatorname{sech} x_{0}, \\
& =\left(\mu-2 \kappa_{\mu}\right) \operatorname{sech}^{2} x_{0}-\kappa_{\mu} x_{0}^{2}+O\left(x_{0}^{4}\right) .
\end{aligned}
$$

A negative dipole strength $\kappa_{\mu}$, which corresponds to the induced supercurrent $\varepsilon$ circulating in the direction of decreasing $x$, thus increases the height of the potential barrier at the lithographic microshort.

The order-of-magnitude enhancement of the microshort depinning current evident in Fig. 8(b) is due to the dipole current $\varepsilon$ induced by magnetic field perpendicular to the junction plane. The field is from flux quanta trapped within the electrode rings, possibly in combination with a residual background field $H_{Z 0}$. The background field for the data presented in Figs. 7 and 8 is likely to be different because they were recorded in different cryostats. An increase in the depinning current due to trapped flux takes on discrete values whereas the increase caused by a background field does not and can therefore be much smaller. Another difference in the effect of trapped flux and a background field is that they induce dipole currents $\varepsilon$ of opposite polarity.

\section{B. Enhanced vortex pinning due to magnetic field applied transverse to the junction plane}

We have investigated the effect on the microshort depinning current of a uniform external field $H_{Z}$ applied transverse to the plane of a junction in the superconducting state. The transverse field $H_{Z}$ was generated using a current coil located underneath the chip. Note that, since the field lines produced by the coil are screened by the superconducting electrodes and cannot pass through the hole of the electrode loop, deflected lines concentrate near the outer edges of the electrodes. ${ }^{43,44}$ Thus the magnitude of the local external field tangential to the electrode surface is substantially larger than $H_{Z}$. In the analogous situation of a disk of radius $r$ and thickness $a$ in the Meissner state in a uniform transverse field, the field at the edge of the disk is a factor of $(1+\sqrt{2 r / a})$ greater. $^{45}$ 


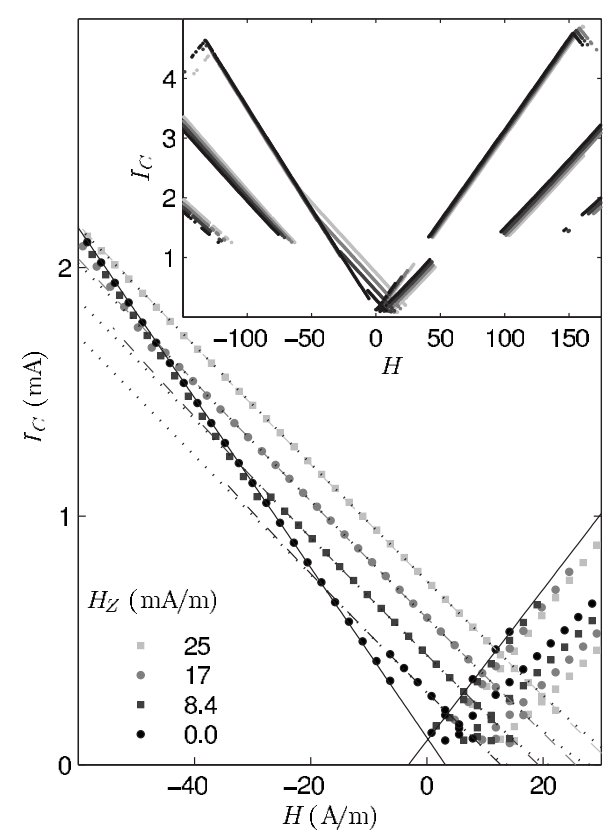

FIG. 10. Vortex-depinning current $I_{C}$ as a function of applied in-plane magnetic field $H$ and perpendicular field $H_{Z}$. Measurements were recorded at a temperature of $T=1.4 \mathrm{~K}$ using junction 1 . The microshort depinning current at $H=0 \mathrm{~A} / \mathrm{m}$ depends linearly on the applied perpendicular field $H_{Z}$. Also plotted is a linear fit (solid line) to the magnetic field depinning current for $H_{Z}=0 \mathrm{~A} / \mathrm{m}$. The fitted depinning current from the microshort barrier is indicated for two cases of misalignment of the solenoid field $H$ : $0.057^{\circ}$ out of (dotted line) and $35^{\circ}$ within (dashed line) the junction plane. The inset displays the $I_{C}(H)$ pattern as a function of $H_{Z}$ over a field $H$ interval which includes the supercurrent maxima.

Figure 10 displays the $I_{C}(H)$ patterns recorded for various perpendicular field strengths $H_{Z}$ applied to the same initial single-vortex junction state, which was obtained by cooling in zero field. An offset in the in-plane field $H$, due to deflection of the applied transverse field $H_{Z}$ through the tunnel barrier, has been removed from each $I_{C}(H)$ pattern. The approximately straight lines of the patterns are associated with the vortex escaping over a potential barrier due to either the magnetic field $H$, the microshort, or the injectors as indicated in Fig. 7 for zero perpendicular field $H_{Z}$. The measured microshort depinning current clearly increases with increasing magnitude of the circulating supercurrent induced by the perpendicular field.

The dipole strength $\kappa_{\mu}$ depends on the applied transverse field $H_{Z}$ as $\kappa_{\mu}=-\zeta\left(H_{Z}+H_{Z 0}\right)$ where $\zeta$ is a proportionality constant. For small $\kappa_{\mu}$ or $x_{0}$, Eq. (48) approximates to $U^{\mu}\left(x_{0}\right)=\bar{\mu} \operatorname{sech}^{2} x_{0}$ where $\bar{\mu}$ is an effective microshort strength that varies linearly with the transverse field $H_{Z}$

$$
\bar{\mu}=\mu+2 \zeta\left(H_{Z}+H_{Z 0}\right) .
$$

With this assumption, we fitted the microshort depinning current to Eq. (22) and determined the magnitude of the background perpendicular field $H_{Z 0}=0.011 \mathrm{~A} / \mathrm{m}$ and the constant $\zeta=8 \mathrm{~m} / \mathrm{A}$ using the method of least squares. An additional fit parameter was the degree of misalignment of the solenoid field $H$.
The measured microshort depinning current depends more strongly on the in-plane field $H$ than expected from Eq. (22) as is seen from Fig. 7. We believe that this was caused by a misalignment of the solenoid field $H$. As is shown in Fig. 10, a misalignment of either $\theta_{1}=0.057^{\circ}$ out of (dotted lines) or $\theta_{2}=35^{\circ}$ within (dashed lines) the junction plane fits the observed $H$ dependence of the microshort depinning current. In both cases, one fit curve is plotted for each of the four values of perpendicular field $H_{Z}$ under which $I_{C}(H)$ patterns were recorded. That the gradient of the measured microshort $I_{C}(H)$ curve depends so sensitively on the degree of misalignment out of the junction plane results from the solenoid field $H$ having a $z$ component when directed out of the plane. The effective microshort strength $\bar{\mu}$ is then a function of this component $H \sin \theta_{1}$ as well as the intentionally applied perpendicular field $H_{Z}$. The larger than predicted gradient of the microshort $I_{C}(H)$ curve could also be due to a simultaneous misalignment of the solenoid field $H$ both within and out of the junction plane. Hence the discrepancy seen in Fig. 7 between the measured microshort depinning current and the prediction of the one-dimensional model, given by Eq. (22), can be attributed to a misalignment of the solenoid field $H$ in addition to the presence of a small background perpendicular field $H_{Z 0}=0.011 \mathrm{~A} / \mathrm{m}$. One means of avoiding interaction between the lithographic microshort and transverse field is described in the next section.

\section{Decoherence sources and improved design}

The vortex qubit has been predicted to have a long coherence time for a superconducting qubit, on the order of tens of microseconds. ${ }^{10}$ This estimate considered the effect of quasiparticle dissipation and weakly fluctuating critical and bias currents in a long linear junction. Low-frequency criticalcurrent noise was found to lowest order not to alter the shape of the vortex potential and hence not to contribute to decoherence. Since the equilibrium density of quasiparticles is exponentially small at low temperatures, the coherence time was predicted to be limited by low-frequency bias noise.

Additional factors which could reduce the coherence time in our proposed qubit are increased sensitivity to flux noise, the presence of excess quasiparticles, and interaction with two-level systems in the dielectric. Flux fluctuations in the $y$ or $z$ directions modulate the barrier height of the microshort qubit, causing exponential change in the tunneling frequency. Therefore it is important to minimize flux noise. Excess quasiparticles are generated when the junction switches to the voltage state. Their contribution to shot noise in the bias current can be decreased with quasiparticle traps. ${ }^{46}$ Twolevel systems are thought to be a major source of decoherence in superconducting qubits. ${ }^{47}$ They originate from charge fluctuations and couple to the qubit via the electric field $\dot{\varphi} / t_{j}$ (Ref. 48) where $t_{j}$ is the tunnel-barrier thickness. In a microshort qubit, two-level systems near the microshort may couple to the qubit via the phase oscillation which results when the vortex oscillates between the $|L\rangle$ and $|R\rangle$ states. The amplitude of the phase oscillation is largest at the microshort and decays over a few Josephson lengths. The number of two-level systems which interact with the microshort qubit 
could be reduced by employing a tunnel barrier of $a-\mathrm{Si}: \mathrm{H},{ }^{49}$ $\mathrm{SiN}_{x},{ }^{47}$ or epitaxial $\mathrm{Al}_{2} \mathrm{O}_{3} \cdot{ }^{50}$

Decoherence due to flux noise transverse to the junction plane is avoided in a microshort qubit without an electrode loop. For example, an improved qubit design is a long junction which consists of two linear segments separated by an annular segment that contains the lithographic microshort. At the place where the junction centerline transitions from annular to straight, the field-induced potential barrier levels off as seen from Eq. (6) because, in the straight segments, the component $h_{R}$ of the in-plane field transverse to the junction centerline is constant. The location of the transition is chosen so that, at the readout field, the microshort depinning current $\gamma_{C 2}$ is greater than the field depinning current $\gamma_{C 1}$. This enables state detection with an RS flip flop at one end of the junction ${ }^{14}$ provided that the readout bias permits the vortex to overcome the field-induced potential barrier but not the microshort-induced barrier. At the other end of the junction is a SFQ generator for qubit initialization. ${ }^{14}$ As well as insensitivity to transverse field noise, this design offers the advantage of faster readout, during which fewer excess quasiparticles are generated. Also, the length of the junction no longer constrains the radius of the annular part; by choosing a shorter radius $r$, control pulses of a given amplitude $\Delta \bar{h}$ are achieved with smaller amplitude pulses of the in-plane field $H$. Using in-plane field pulses large enough that the potential transforms from a double to a single well could reduce the sensitivity of the coherent oscillation frequency to flux fluctuations in the $y$ direction. ${ }^{51}$

\section{CONCLUSION}

We have studied a vortex qubit based on an annular Josephson junction containing a lithographic microshort. From the one-dimensional vortex potential, we have derived the magnetic field dependence of the vortex-depinning current over a microshort-induced potential barrier. We have also obtained the frequency of vortex oscillation within the well. From this we find that, for a low microshort-induced potential barrier, the exponent in the coupling $\Delta_{0}$ between degenerate minima varies with field as $(1-\bar{h})^{3 / 2}$.

The proposed vortex qubit design has been tested experimentally in the classical regime. We observed bistable vortex states located on either side of the microshort. Preparation of the vortex in a given potential well was achieved by means of a shaker sequence of bias-current pulses. We noticed that the depinning current from a lithographic microshort can be enhanced by flux trapped around both superconducting electrodes as well as field applied perpendicular to the junction plane.

\section{ACKNOWLEDGMENTS}

We thank Edward Goldobin for valuable discussions. This work was partially supported by the "European Superconducting Quantum Information Processor" (EuroSQIP) project, the European Science Foundation Research Networking Programme "Arrays of Quantum Dots and Josephson Junctions" (AQDJJ), the Deutsche Forschungsgemeinschaft, and the EPSRC under Grant No. EP/E042589/1.
${ }^{1}$ Y. Nakamura, Y. A. Pashkin, and J. S. Tsai, Nature (London) 398, 786 (1999).

${ }^{2}$ J. M. Martinis, S. Nam, J. Aumentado, and C. Urbina, Phys. Rev. Lett. 89, 117901 (2002).

${ }^{3}$ I. Chiorescu, Y. Nakamura, C. J. P. M. Harmans, and J. E. Mooij, Science 299, 1869 (2003).

${ }^{4}$ D. Vion, A. Aassime, A. Cottet, P. Joyez, H. Pothier, C. Urbina, D. Esteve, and M. H. Devoret, Science 296, 886 (2002).

${ }^{5}$ A. Wallraff, Y. Koval, M. Levitchev, M. V. Fistul, and A. V. Ustinov, J. Low Temp. Phys. 118, 543 (2000).

${ }^{6}$ A. Kemp, A. Wallraff, and A. V. Ustinov, Phys. Status Solidi B 233, 472 (2002).

${ }^{7}$ M. V. Fistul and A. V. Ustinov, Phys. Rev. B 68, 132509 (2003).

${ }^{8}$ P. D. Shaju and V. C. Kuriakose, Phys. Lett. A 332, 326 (2004).

${ }^{9}$ A. Wallraff, Ph.D. thesis, Universität Erlangen-Nürnberg, 2001.

${ }^{10}$ J. H. Kim, R. P. Dhungana, and K.-S. Park, Phys. Rev. B 73, 214506 (2006).

${ }^{11}$ D. W. McLaughlin and A. C. Scott, Phys. Rev. A 18, 1652 (1978).

${ }^{12}$ P. D. Shaju and V. C. Kuriakose, Physica C 424, 125 (2005).

${ }^{13}$ T. Kato and M. Imada, J. Phys. Soc. Jpn. 65, 2963 (1996).

${ }^{14}$ V. K. Kaplunenko and A. V. Ustinov, Eur. Phys. J. B 38, 3 (2004).

${ }^{15}$ G. Carapella, F. Russo, R. Latempa, and G. Costabile, Phys. Rev. B 70, 092502 (2004).
${ }^{16}$ P. D. Shaju and V. C. Kuriakose, Physica C 434, 25 (2006).

${ }^{17}$ E. Goldobin, A. Sterck, and D. Koelle, Phys. Rev. E 63, 031111 (2001).

${ }^{18}$ A. Benabdallah, J. G. Caputo, and A. C. Scott, Phys. Rev. B 54, 16139 (1996).

${ }^{19}$ A. Kemp, Ph.D. thesis, Universität Erlangen-Nürnberg, 2006.

${ }^{20}$ A. Davidson, B. Dueholm, B. Kryger, and N. F. Pedersen, Phys. Rev. Lett. 55, 2059 (1985).

${ }^{21}$ A. Shnirman, E. Ben-Jacob, and B. Malomed, Phys. Rev. B 56, 14677 (1997).

${ }^{22}$ A. Wallraff, A. Lukashenko, J. Lisenfeld, A. Kemp, M. Fistul, Y. Koval, and A. V. Ustinov, Nature (London) 425, 155 (2003).

${ }^{23}$ J. R. Friedman, V. Patel, W. Chen, S. K. Tolpygo, and J. E. Lukens, Nature (London) 406, 43 (2000).

${ }^{24}$ C. H. van der Wal, A. C. J. ter Haar, F. K. Wilhelm, R. N. Schouten, C. J. P. M. Harmans, T. P. Orlando, S. Lloyd, and J. E. Mooij, Science 290, 773 (2000).

${ }^{25}$ Y. Makhlin, G. Schön, and A. Shnirman, Nature (London) 398, 305 (1999).

${ }^{26}$ A. N. Price, A. Kemp, and A. V. Ustinov (unpublished).

${ }^{27}$ N. Grønbech-Jensen, P. S. Lomdahl, and M. R. Samuelsen, Phys. Lett. A 154, 14 (1991).

${ }^{28}$ N. Grønbech-Jensen, P. S. Lomdahl, and M. R. Samuelsen, Phys. Rev. B 43, 12799 (1991).

${ }^{29}$ U. Weiss, Quantum Dissipative Systems, 2nd ed. (World Scien- 
tific, Singapore, 1999)

${ }^{30}$ A. J. Leggett, S. Chakravarty, A. T. Dorsey, M. P. A. Fisher, A. Garg, and W. Zwerger, Rev. Mod. Phys. 59, 1 (1987).

${ }^{31}$ A. A. Abdumalikov, V. V. Kurin, C. Helm, A. De Col, Y. Koval, and A. V. Ustinov, Phys. Rev. B 74, 134515 (2006).

${ }^{32}$ Hypres Inc, Elmsford, NY 10523, U.S.A., http:// www.hypres.com

${ }^{33}$ A. Franz, A. Wallraff, and A. V. Ustinov, J. Appl. Phys. 89, 471 (2001).

${ }^{34}$ A. Wallraff, A. Lukashenko, C. Coqui, A. Kemp, T. Duty, and A. V. Ustinov, Rev. Sci. Instrum. 74, 3740 (2003).

${ }^{35}$ A. V. Ustinov, Appl. Phys. Lett. 80, 3153 (2002).

${ }^{36}$ B. A. Malomed and A. V. Ustinov, Phys. Rev. B 69, 064502 (2004).

${ }^{37}$ N. Martucciello and R. Monaco, Phys. Rev. B 53, 3471 (1996).

${ }^{38}$ V. Lefevre-Seguin, E. Turlot, C. Urbina, D. Esteve, and M. H. Devoret, Phys. Rev. B 46, 5507 (1992).

${ }^{39}$ A. N. Price et al. (unpublished).

${ }^{40}$ R. Monaco, M. Aaroe, J. Mygind, R. J. Rivers, and V. P. Koshelets, Phys. Rev. B 77, 054509 (2008).

${ }^{41}$ E. Goldobin, N. Stefanakis, D. Koelle, and R. Kleiner, Phys. Rev. B 70, 094520 (2004).

${ }^{42}$ E. Goldobin, A. Sterck, T. Gaber, D. Koelle, and R. Kleiner,
Phys. Rev. Lett. 92, 057005 (2004).

${ }^{43}$ R. Monaco, M. Aaroe, J. Mygind, and V. P. Koshelets, J. Appl. Phys. 102, 093911 (2007).

${ }^{44}$ R. Monaco, M. Aaroe, J. Mygind, and V. P. Koshelets, J. Appl. Phys. 104, 023906 (2008).

${ }^{45}$ M. Benkraouda and J. R. Clem, Phys. Rev. B 53, 5716 (1996).

${ }^{46}$ K. M. Lang, S. Nam, J. Aumentado, C. Urbina, and J. M. Martinis, IEEE Trans. Appl. Supercond. 13, 989 (2003).

${ }^{47}$ J. M. Martinis, K. B. Cooper, R. McDermott, M. Steffen, M. Ansmann, K. D. Osborn, K. Cicak, S. Oh, D. P. Pappas, R. W. Simmonds, and C. C. Yu, Phys. Rev. Lett. 95, 210503 (2005).

${ }^{48}$ I. Martin, L. Bulaevskii, and A. Shnirman, Phys. Rev. Lett. 95, $127002(2005)$.

${ }^{49}$ A. D. O'Connell, M. Ansmann, R. C. Bialczak, M. Hofheinz, N. Katz, E. Lucero, C. McKenney, M. Neeley, H. Wang, E. M. Weig, A. N. Cleland, and J. M. Martinis, Appl. Phys. Lett. 92, 112903 (2008).

${ }^{50}$ S. Oh, K. Cicak, J. S. Kline, M. A. Sillanpää, K. D. Osborn, J. D. Whittaker, R. W. Simmonds, and D. P. Pappas, Phys. Rev. B 74, 100502(R) (2006)

${ }^{51}$ S. Poletto, F. Chiarello, M. G. Castellano, J. Lisenfeld, A. Lukashenko, C. Cosmelli, G. Torrioli, P. Carelli, and A. V. Ustinov, New J. Phys. 11, 013009 (2009). 